\title{
11 Das Sein als Schein (I): Die tragische Zuspitzung der Pendelbewegung und die iterativ-regenerativen Ansprüche des „Äußerlichen überhaupt“
}

In Teil 1 des „Schein“-Abschnitts benennt Hegel in aller wünschenswerten Klarheit den vorausgesetzten historischen Horizont, auf den seine Kritik des Scheins zielt, d.h. er benennt den äußerlich-genetischen Grund des Scheinbegriffs. Dergestalt zielt die Analyse des Scheins nämlich auf eine fundamentalbegriffliche Kritik des Skeptizismus und des neueren Idealismus (Leibniz, Kant, Fichte), insofern diese mit einem (je verschieden ausgelegten) Konzept von Erscheinungshaftigkeit (Phänomenalität) operieren (GW 11, S. $246 \mathrm{f}$.). Dieses aber ist laut Hegel durch einen fundamentalen, im negativen Sinn wirksamen, also selbstzerstörerischen Widerspruch gekennzeichnet, der an sich bereits die Unhaltbarkeit der mit ihm verbundenen Positionen manifest machen soll:

\footnotetext{
So ist der Schein [...] überhaupt nicht ein gleichgültiges Seyn, das ausser seiner Bestimmtheit und Beziehung auf das Subject wäre. [...] [J]ener Schein sollte überhaupt keine Grundlage eines Seyns haben, in diese Erkenntnisse sollte nicht das Ding-an-sich eintreten. Zugleich aber ließ der Sceptizismus mannigfaltige Bestimmungen seines Scheins zu, oder vielmehr sein Schein hatte den ganzen mannigfaltigen Reichthum der Welt zum Inhalte. Eben so begreift die Erscheinung des Idealismus den ganzen Umfang dieser mannichfaltigen Bestimmtheiten in sich. Jener Schein und diese Erscheinung sind unmittelbar so mannichfaltig bestimmt. (GW 11, S. 246f.)
}

Hegel macht deutlich, dass bezüglich der historischen Positionen, die mit dem Scheinbegriff gearbeitet haben, die Kritik des Scheins eine totale sein muss. Mit ihm ist nämlich in historischer Perspektive ein Denken kritisiert, das die Welt als Erscheinung a) nur in Bezug auf ein Subjekt denkt, welches Konstitutionsgrund dieser Erscheinungen ist, weshalb zugleich b) dem so gearteten Schein keine Beziehung auf das Wesentliche der „Dinge an sich“ unabhängig vom Subjekt zukommt. Zugleich aber wird von diesen Positionen dieser Schein behandelt wie ein selbstständiges und an sich substanzielles Sein, d.h. als „ein gegebener Inhalt“ (GW 11, S. 247) in unmittelbarer und mannigfaltiger Bestimmtheit, d.h. als ein mehr denn bloß trügerisches Gegebensein von Realität. In der Kritik des Scheinbegriffs erweist sich, so hat Christian Iber pointiert formuliert, „der Subjektivismus des Skeptizismus und vor allem des neueren Idealismus [...] als ein 
sich nicht durchschauender ontologischer Realismus“"75, weil er - vor der Folie einer Verdopplung des Seienden in Wesen und Erscheinung - der Erscheinung der Form nach keinen substanziellen Inhalt mehr zugesteht, zugleich aber diese noch wie ein substanziell unmittelbares Sein behandelt. Mit anderen Worten: Wenn die Welt der Erscheinungen nur vom Subjekt konstituiert ist, so kann sie mit der Wesentlichkeit der Dinge an sich nichts zu tun haben ${ }^{76}$; zugleich jedoch behandelt das idealistische Subjekt diese Erscheinungswelt wie einen substanziellen, unmittelbaren und wahrhaften Inhalt. Hegel reformuliert damit die schon von Kant explizit gemachte Spannung, dass die Semantik von „Erscheinung“ stets transitiv als transzendierend $\mathrm{zu}$ denken ist, also als Erscheinung von etwas Anderem (als die Erscheinung selbst) konzeptualisiert werden muss, um nicht logisch zu implodieren, zugleich jedoch die transzendentalidealistische kantische Systemarchitektur eben diesen Darstellungscharakter des Wesentlichen in der und als Erscheinung bestreitet (vgl. Kap. 1.1.5 im Hauptteil I), als offenen und destruktiven Widerspruch. Jede Erscheinung von etwas bezieht sich notwendig auf etwas Anderes als sie selbst, dessen Erscheinung sie darstellt; zugleich aber tritt dieses Andere in der Erscheinung sich selbst gegenüber als ein Anderes auf, weil es nicht wie es an sich ist, sondern nur wie es erscheint, in der Erscheinung manifest wird. ${ }^{77}$ Dem Schein eignet so nach Hegel eine seltsame destabilisierende Zwischenstellung in Bezug auf seinen Unterschied zum Wesen: Einerseits liegt das Wesen beziehungslos zu ihm hinter ihm verborgen und degradiert damit den Schein zu einem unhaltbaren, substanz- und wahrheitslosen Trugbild. Zugleich jedoch bewahrt andererseits der Schein eine Art von ontologischer Standfestigkeit, die ihm in der Form des Vorgängers vom Wesen, dem Sein, einen eigenen substanziellen Gehalt unmittelbar zuspricht, durch welchen er sein Bestehen

75 Iber: Metaphysik absoluter Relationalität, S. 81.

76 Wenn dies auch eine problematische Kant-Lesart Hegels ist (vgl. Kap. 1 im Hauptteil I), so trifft sie doch zumindest den Kern eines Problems, nämlich das fehlende objektive Kriterium für die Wahrhaftigkeit dieser Erscheinungen.

77 Niklas Luhmann wird später diese Aporie in seiner Systemtheorie wiederholen, indem er die Kommunikation zwischen Systemen insofern quasi als „autistisch“ charakterisiert, als jedes Eindringen von systemexternen Informationen in das System nur durch und in der Grammatik des Systems und damit nicht als wirklich anderer Inhalt möglich ist (vgl. Niklas Luhmann: Soziale Systeme. Grundriß einer allgemeinen Theorie, Frankfurt am Main 1985, S. 183). Claus-Artur Scheiers Kritik an Luhmann geht in eine ähnliche Richtung, wenn er diesem eine „philosophische Hilflosigkeit“ hinsichtlich der „eigenen Leitdifferenz“ attestiert (Claus-Artur Scheier: „Differenz der Hegelschen und Luhmannschen Philosophie des Systems“, in: Idee, Geist, Freiheit. Hegel und die zweite Natur, hg.v. Wolfgang Neuser/Pirmin Stekeler-Weithofer, Würzburg 2017, S. 225-236, hier: S. 236): „Zwar sind Strukturen reversible Sedimente von Operationen, aber nur innerhalb des Systems, nicht des operierenden Systems selbst.“. 
erhält. Es ist dieser Rückfall des Substanzlosen an sich in die äußerliche, seinem Inhalt gegenüber gänzlich unangebrachte Form seinshaftiger Substanzialität im Schein, mit anderen Worten sein Bestehen als die völlige Äußerlichkeit seines Inhalts und seiner Form gegeneinander als kategorialer Ausdruck eines unwahren Bestehens überhaupt, welche den Schein scheinbar als zentrale Gedankenbestimmung des Falschen schlechthin auszeichnet und brandmarkt. Michael Theunissens Interpretation der Logik hat denn auch von diesem historisch-genetischen Aspekt des Scheins ihren Ausgang genommen und völlig nachvollziehbar den Schein sowohl als nicht nur auf diesen Abschnitt beschränktes kritisches Prinzip der gesamten Logik verstanden, als auch vom Schein aus den geschichtlichen bzw. sozialgeschichtlichen kritischen Aspekt der Logik mit Blick auf Marx begründet. ${ }^{78}$

Man wird allerdings den argumentativen Möglichkeiten des Scheinbegriffs bzw. des Scheinkapitels nicht vollständig gerecht, wenn man diesen historischkritischen Aspekt, der anscheinend in die bloße Negativität totaler Kritik und Ablehnung alles Scheinhaften führt, nicht unterscheidet von dem im engeren Sinn kategorialen, nämlich im Fortgang des Wesens sozusagen argumentationsfunktionalen Aspekt einer rein logischen Genese des Begriffs. Unter diesem Aspekt aber geht der Schein nicht aus konkreten historischen Konfigurationen der Philosophiegeschichte hervor und bildet deren Inbegriff des Falschen, sondern er nimmt eine notwendige Stelle innerhalb des rein logischen Entwicklungszusammenhangs eines reinen Begriffs von „Realsein überhaupt“ im Raum des Übergangs vom Kategorienfeld des Seins zum Kategorienfeld des Wesens ein. Und in dieser vollständig intralogischen Genese kommen ihm wiederum positive Wahrheits- und Funktionspotenziale zu, die sich über die bloße Kritik eines kategorialen Abdrucks falschen Bewusstseins nicht erfassen oder gar erklären lassen, wenngleich beide natürlich argumentativ im Scheinkapitel in engen Wechselwirkungen stehen. Uns soll im Folgenden nur dieser zweite, rein intralogische, d.h. eben kategoriale, positive Aspekt des Scheins näher interessieren.

Das Seyn ist Schein. Das Seyn des Scheins besteht allein in dem Aufgehobenseyn des Seyns, in seiner Nichtigkeit; diese Nichtigkeit hat es im Wesen, und ausser seiner Nichtigkeit, ausser dem Wesen ist es nicht. Er ist das Negative gesetzt als Negatives.

Der Schein ist der ganze Rest, der noch von der Sphäre des Seyns übrig geblieben ist. Er scheint aber selbst noch eine vom Wesen unabhängige unmittelbare Seite zu haben und ein Anderes desselben überhaupt zu seyn. Das Andere enthält überhaupt die zwey Momente des Daseyns und des Nichtdaseyns. Das Unwesentliche, indem es nicht mehr ein Seyn hat, so bleibt ihm vom Andersseyn nur das reine Moment des Nichtdaseyns; der Schein ist diß un mittel ba re Nichtdaseyn so in der Bestimmtheit des Seyns, daß es nur in

78 Vgl. Theunissen: Sein und Schein, S. 9. 
der Beziehung auf anderes, in seinem Nichtdaseyn Daseyn hat; das Unselbständige, das nur in seiner Negation ist. Es bleibt ihm also nur die reine Bestimmtheit der Unmittelbarkeit; es ist als die reflectirte Unmittelbarkeit, das ist, welche nur vermittelst ihrer Negation ist, und die ihrer Vermittlung gegenüber nichts ist, als die leere Bestimmung der Unmittelbarkeit des Nichtdaseyns. (GW 11, S. 246)

Dieser ungemein dichte Abschnitt enthält die rein intralogische Essenz des Scheinbegriffs in seinen Anfangsbedingungen. Hegel stellt sich hier die Frage, wie eine Bestimmung wie der Schein ein Bestehen haben kann, d.h. einen Geltungsanspruch vertritt, obwohl der Schein keinerlei Berechtigung mehr zu haben scheint, im Raum des Wesens wirksam zu sein. Kernbegriffe dieses Abschnitts sind dabei die Charakterisierungen „unmittelbare[s] Nichtdasein“ und „reflektierte Unmittelbarkeit“ für den Schein. Mit ihnen beschreibt Hegel den nun erreichten Extrempunkt der bereits erörterten Pendelbewegung des Übergangs vom Sein zum Wesen: d.h. die äußerste Art des Verhältnisses, in welchem das bereits vollständig im Wesen aufgehobene Sein sich erneut als Anderes zum Wesen, d. h. in seiner (logisch) früheren Gestalt, geltend machen kann und so eben jene bereits untergegangene logische Gestalt des Seins restituiert, die auf dieser Stufe gerade keine Geltung mehr zu beanspruchen vermag. Allerdings ist diese logische Gestalt des Seins im Schein in verschiedenen Hinsichten so brüchig geworden, dass ihr Bestehen auf einem vollständig paradoxen Konstitutionsakt beruht: Das Sein ist nun auf dieser Stufe der Wiederkehr in seiner ,alten' logischen Substanz derart aufgezehrt, dass es ein Bestehen gegen das und neben dem Wesen nur noch auf paradoxe Weise - nämlich als ein rein Negatives - bewerkstelligen kann. Im Schein „scheint“ das Sein nur noch „eine vom Wesen unabhängige unmittelbare Seite zu haben und ein A nderes desselben überhaupt zu seyn“ (GW 11, S. 246): D.h. die Eigenständigkeit des Seins und damit die seinslogische Grammatik des Andersseins überhaupt überlebt in ihm scheinbar nur noch als Lüge, d.h. als reine „Nichtigkeit“ des Geltungsanspruchs, die Form und den Grund des reinen Denkens abzugeben. Im Schein ist die vollständige Aufhebung der Logik des Seins in die Logik des Wesens bereits ein so vollständig anerkannter Fakt der Gedankenbestimmung des Scheins selbst, dass ihr Gehalt einzig darin besteht, sich selbst $\mathrm{zu}$ bestreiten und als dieses Bestreiten zu bestehen: Schein bedeutet im reinen intralogischen Sinn bei Hegel, dass die vollständige Negation und Aufhebung des Seins im Wesen als eine Bestimmung gegen das Wesen besteht, also der Form nach seinslogisch eben das sich verwirklicht, was im Gehalt wesenslogisch vollständig bestritten wird. Der Schein gehört als reine Kategorie des aufs äußerste angespannten Übergangs vom Sein zum Wesen am konsequentesten beiden logischen Sphären an, der Sphäre des Seins und der Sphäre des Wesens, weil beide im Schein über die Äußerlichkeit von Inhalt (Wesen) und Form (Sein) 
auseinandertreten und zugleich untrennbar aufeinander bezogen sind. Deshalb ist der Schein dem Aspekt seines Gehalts nach „unmittelbare[s] Nichtdaseyn“ (GW 11, S. 246): Denn das Sein existiert im Schein nur in der Bestimmung seiner vollständigen Negation (Nichtdasein), aber zugleich in der Form unmittelbaren Daseins. Das Sein besteht im Schein gerade so, wie es dem Inhalt des Scheins nach als vollständig falsch erörtert wird: Es ist so der vollendete Widerspruch von Gehalt und Form. Der Schein hat zu sich selbst ein ironisches Verhältnis: Er besteht als absolute logische Ironie. Die „Nichtigkeit“ des Seins, „das Negative gesetzt, als Negatives“ (GW 11, S. 246) im Schein bedeutet zugleich eine radikale Selbstbestreitung des Scheins als ein erstes Verhältnis selbstbezüglicher Negativität: Da er der Form nach (als unmittelbares Dasein gegen das Wesen) dem Sein in seiner unaufgehobenen Form zugehört, die er zugleich seinem Gehalt nach als bereits vollständig im Wesen verschwunden und damit ohne jede Möglichkeit der weiteren Geltung beschreibt, ist er seiner Form nach „reflectirte Unmittelbarkeit“ (GW 11, S. 246). Damit bezeichnet Hegel eine Unmittelbarkeit, „welche nur vermittels ihrer Negation ist“ (GW 11, S. 246), d.h. die als an sich unmittelbar negierte besteht. In dieser Hinsicht ist der Gehalt des Scheins der Form des Scheins nicht mehr nur äußerlich entgegengesetzt, sondern affiziert sie tiefgreifend und fällt mit ihr zusammen: Das Bestreiten des Seins im Gehalt des Scheins führt dazu, dass auch die seinshafte Form des Scheins selbst nur als negierte gedacht werden kann. Wo der Schein dem Inhalt nach aussagt, dass das Sein keinerlei Bestehen mehr hat, und der Schein trotzdem in dieser Form des Seins existiert, da kann diese Existenzform des Scheins keine ungebrochene mehr sein. Genauer besehen, überlagern sich also im Schein zwei wiederum gegensätzliche Verhältnisse von Form und Inhalt, an sich selbst vollzogener und beschriebener kategorialer Grammatik, ohne zu einer integrativen Form zu finden: Vielmehr buchstabieren sie eher ein Verhältnis aus, das man mit Hölderlin ,geradentgegengesezt“ nennen könnte ${ }^{79}$, weil in ihm die sich eigentlich auslöschenden

79 Das große Fragment Wenn der Dichter einmal des Geistes mächtig ist ... (Die Verfahrensweise des poetischen Geistes) darf als zentraler Text zu Hölderlins Dialektik angesehen werden. In ihm durchdenkt Hölderlin spekulative Begriffe wie „Geist“, „Form“, „Materie“, „Einheit“, „Gegensatz“, „Bedeutung“ und „Ich“ stets in der Weise, dass sich diese Begriffe zueinander in konstitutive Gegensatzverhältnisse setzen, innerhalb derer sie sich erst erhalten und entfalten können. Zudem sind die Gegensätze dabei stets so gedacht, dass sie sich jeweils nur in der stabilen Form der Entgegensetzung auch mit sich selbst zusammenschließen können, weil durch die Gegensetzung stets zugleich im Anderen, Entgegengesetzten das Eigene mitgesetzt ist. Wo Bestimmungen folglich den Punkt ihrer Entgegensetzung erreicht haben, treffen sie im widersprechenden Anderen immer zugleich auch auf sich als Anderes, weil das Entgegengesetzte eben durch die Beziehung auf sein Gegenteil bestimmt ist und damit das Andere in sich hineingenommen hat. Die Form des Gegensatzes bedingt für Hölderlin eine Logik des Wechsels, in der die 
Entgegengesetzten im Entgegensetzen zugleich auch gleich sind. So sind sie im Anderen bei sich, ohne allerdings dadurch im hegelschen Sinn mit diesem eine neue Einheit zu bilden: Der „Widerstreit“ (Friedrich Hölderlin: „Wenn der Dichter einmal des Geistes mächtig ist ...“, in: Ders.: Theoretische Schriften, hg.v. Johann Kreuzer, Hamburg 1998, S. 39-62, hier: S. 39) ist mithin die „Art, wie eines in sich selbst zusammenhängt“ (Friedrich Hölderlin: „Pindar-Fragmente“, in: Ders.: Theoretische Schriften, hg.v. Johann Kreuzer, Hamburg 1998, S. 111-118, hier: S. 113). Die Entgegengesetzten okkupieren so zuweilen die Funktion des Anderen, ohne ihre Entgegensetzung aufzugeben; d. h. sie wirken als das Andere im strikten Gegensatz zum Anderen und zeigen sich so als durchgängig miteinander verflochten, was für Hölderlin die einzige Möglichkeit eines echten „Ganzen“, die „absolute Form einer Einheit“ (Friedrich Hölderlin: „Seyn, Urtheil“, in: Ders.: Theoretische Schriften, hg.v. Johann Kreuzer, Hamburg 1998, S. 7-9, hier: S. 7) darstellt. Die widersprechenden Forderungen des Geistes nach Einigkeit/Zugleich/Ewigkeit und nach Mannigfaltigkeit/Wechsel/Fortschritt müssen in seinen Manifestationen in jedem Moment zugleich befriedigt werden. Folglich werden der „Widerstreit“ des Geistes mit sich und das Gesetz der Selbstentgegensetzung des Wirklichen zwischen Endlichkeit und Unendlichkeit, Wechsel und Ewigkeit zu den grundlegenden Normen von Hölderlins Denken. Exemplarisch kann dafür der Begriff der „Bedeutung“ (Hölderlin: Wenn der Dichter einmal des Geistes mächtig ist, S. 42) stehen, den Hölderlin als dialektischen Vereinigungspunkt zwischen den Gegensätzen von Vergeistigung und Materialisierung begreift: aber so, dass sie „zwischen beiden“ steht und „sich selber überall entgegengesezt ist“, weil sie alles „entgegengesezte vergleicht, alles einige trennt“ (Hölderlin: Wenn der Dichter einmal des Geistes mächtig ist, S. 43). „Bedeutung“ im hölderlinschen Sinne vereinigt also die Gegensätze darin, dass der Metagegensatz von Trennen und Vereinigen in ihr zu sich selbst in Gegensatz tritt: indem sie nämlich durch Trennen vereinigt und durch Vereinigen trennt. Möglich wird diese komplizierte reflexive Denkfigur dadurch, dass Hölderlin zwei Begriffe des Entgegensetzens unterscheidet: das „Geradentgegengesetzte“ vom „Harmonischentgegengesetzten“ (Hölderlin: Wenn der Dichter einmal des Geistes mächtig ist, S. 43). Wo das Geradentgegengesetzte durch die ausschließliche und extreme Beziehung des Gegensatzes bestimmt ist, greifen im Harmonischentgegengesetzten die Gegensätze zugleich im Verhältnis der Identität ineinander: und zwar, indem die dem Gehalt nach Entgegengesetzten zugleich in „der Richtung und Grade der Entgegensezung“ (Hölderlin: Wenn der Dichter einmal des Geistes mächtig ist, S. 44) übereinstimmen. Hölderlin unterscheidet hier also am Gegensatz eine Inhalts- von einer Formebene, auf die sich die Bestimmungen von Differenz (Inhalt) und Identität (Form) verteilen. In der Form des Entgegensetzens, d. h. in der formalen Symmetrie des Gegensatzes, entsprechen sich die inhaltlich Widerstreitenden. Von diesen logischen Grundbestimmungen aus entwirft Hölderlin dann eine Logik des Geistes als „poetische Reflexion“ (Hölderlin: Wenn der Dichter einmal des Geistes mächtig ist, S. 45), der sich nur in immer komplizierteren Gegensatz- und Wechselverhältnissen von formaler bzw. materialer Vereinigung und Entgegensetzung erst erhalten und entfalten kann. Als „Entgegensezung welche sich selbst entgegengesezt ist“ (Hölderlin: Wenn der Dichter einmal des Geistes mächtig ist, S. 48), weil sie als und durch ihre Entgegensetzungen ihre Einheit mit sich verwirklicht, darf der Geist weder den Wechsel in der Einheit noch die Einheit im Wechsel verschwinden lassen. Die unendliche Einheit des Geistes, die sich so als ständiger fluktuierender Wechsel zwischen den Aspekten Identität und Gegensatz bildet, kulminiert endlich im letztgründenden harmonischen Widerspruch (Hölderlin: Wenn der Dichter einmal des Geistes mächtig ist, S. 47) zwischen den Perspektiven des „Lebens überhaupt“ (wo der Geist material entgegensetzend und formal vereinigend opperiert) und der „Einigkeit überhaupt“ (wo der Geist material vereinigend und formal entgegensetzend verfährt), als die der Geist erscheint. 
Ähnliche Theorien dialektischer Entfaltung liefert Hölderlin auch für das poetische Ich und die Form des Tragischen. Ähnlich wie für den Geist entwickelt Hölderlin im zweiten Teil des Fragments eine Theorie des (poetischen) Ich, die auf die Frage nach den letzten Bedingungen von Subjektivität abzielt und ebenfalls die radikale Figur des dialektischen Gegensatzes in das Ich einfügt. In komplizierter Auseinandersetzung mit Fichtes Gedanken eines sich in unmittelbarer Einheit mit sich (1. Grundsatz) und im mittelbaren Gegensatz zu einem Nicht-Ich (2., 3. Grundsatz) setzenden Ich zeigt Hölderlin, dass die harmonische Entgegensetzung des Ich zu einer äußeren Sphäre gleichursprünglich und wechselbedingend mit der inneren Einheit des Ich ist (Hölderlin: Wenn der Dichter einmal des Geistes mächtig ist, S. 50f.). Selbstbewusstsein ist demnach kein selbstsuffizientes Phänomen, das letztlich und primär in der unmittelbaren Einheit mit sich seinen Grund findet, sondern zuerst und wesentlich auf eine äußere Entgegensetzung des Ich zu einem Anderen angewiesen. Die innere harmonische Entgegensetzung des Ich zu sich, die den Kern von Selbstsein ausmacht, existiert nur und erkennt sich einzig, indem sie sich zugleich und gleichursprünglich nach außen reproduziert, d.h. im äußeren Gegensatz zu einem anderen Ich verwirklicht. Hölderlin fasst dies in die Maxime: „Seze Dich mit freier Wahl in harmonische Entgegensezung mit einer äußeren Sphäre, so wie du in dir selber in harmonischer Entgegensezung bist, von Natur, aber unerkennbarerweise so lange du in dir selbst bleibst.“ (Hölderlin: Wenn der Dichter einmal des Geistes mächtig ist, S. 53) Weil die „Realität der Unterscheidung“ die „Realität des Erkennens“ (Hölderlin: Wenn der Dichter einmal des Geistes mächtig ist, S. 53) bedingt, ist das „Alleinseyn“ (Hölderlin: Wenn der Dichter einmal des Geistes mächtig ist, S. 54), ganz wörtlich als die Gründung des Ich im bloß eigenen Sein der fugenlosen Einheit mit sich verstanden, für das Ich keine ontologische und epistemologische Option. Hölderlin liefert so im Gewand einer Dichtungstheorie den zumindest prospektiv umfassenden Versuch, die Bereiche von Ich und Welt, Geist und Natur sämtlich in der dialektischen Logik von harmonischer Gegensätzlichkeit und des dialektischen Wechsels zwischen dem Entgegengesetzten zu begründen. Schließlich überführen Hölderlins Überlegungen zur Philosophie des Tragischen und der Tragödie (Grund zum Empedokles, Sophokles-Anmerkungen) seine Idee der Dialektik nochmals in einen neuen Bereich. Tragisch ist für Hölderlin ein Geschehen, in dem „das Ungeheure, wie der Gott und Mensch sich paart, [...] dadurch sich begreift, daß das gränzenlose Eineswerden durch gränzenloses Scheiden sich reinigt“ (Friedrich Hölderlin: „Sophokles-Anmerkungen“, in: Ders.: Theoretische Schriften, hg.v. Johann Kreuzer, Hamburg 1998, S. 94-110, hier: S. 100). Im Tragischen gerät die maßvolle und harmonische dialektische Entgegensetzung insofern aus dem Gleichgewicht, als in ihr die intensive, übermäßige Einheit des Menschen mit dem Göttlichen an sich selbst umschlägt in die übermäßige, nur noch trennende und zerstörende Entgegensetzung: also indem die schlechten Extreme von Vereinigung und Entzweiung sich berühren und als identisch erweisen. Der tragische Held geht folglich unter, weil er sich der Entgrenzung seiner Endlichkeit im Einswerden mit dem Göttlichen vollständig hingibt und damit nicht nur sich, sondern, als exemplarischer Protagonist einer Epoche, die Gesetze und Ordnungen seiner Zeit mit in die Vernichtung reißt. Freilich ergibt sich gerade hieraus der positive geschichtsphilosophische Sinn tragischer Prozesse im Rückgriff auf das Vaterlands-Fragment: Mit dem tragischen Helden geht eine alte Ordnung unter, damit sich im Übergang das Absolute in der Bildung neuer, besserer Ordnungen verwirklichen kann. Die späten Pindar-Fragmente betonen demgegenüber erneut die besondere Bedeutung einer stabilen, harmonischen, Unterschied und Einheit in beständigem Wechsel angemessenen vermittelnden Seinsordnung als Bedingung von Beständigkeit im Strom der „reißenden Zeit“ (Hölderlin: Sophokles-Anmerkungen, S. 97): weil der Mensch in seiner Schwäche „froh ist da, wo er sich halten kann“ (Hölderlin: Pindar-Fragmente, S. 116). Hölderlins 
Extreme in starker Spannung bestehen bleiben, ohne eine andere integrative bzw. erhaltende Kraft als diese Spannung zu besitzen. Zum einen sind im Schein die seinslogische Form und der wesenslogische Inhalt vollständig äußerlich und negativ zueinander bestimmt, indem der Schein seinshaft gegen das Wesen zu bestehen scheint, Ausdruck der vollständigen Verneinung des Seins zu sein. Zum anderen ist dieses seinshaft-unmittelbare Bestehen des Scheins aber selbst derart inhaltlich affiziert von seinem eigenen wesenslogischen Gehalt der Aufhebung des Seins, d.h. seiner eigenen Geltung unterworfen, dass es einzig gemäß dieses Inhalts als reflektierte Unmittelbarkeit und damit nur in der Verneinung seines Bestehens bestehen kann. Damit besteht der Schein, negativ gesehen, als die reine logische Inkonsequenz an sich: Inkonsequent ist an ihm der Widerspruch von Form und Inhalt, wo die seinslogische Form, ohne jedoch deshalb ihr Bestehen zu verlieren, zugleich auch durch den wesenslogischen Gehalt des Scheins bestimmt wird und so einzig „reflektiert“, d.h. in ihrer Verneinung, besteht. Inkonsequent ist aber auch die Integration von Form und Inhalt, wo dieses reflektierte Bestehen seinslogischer Unmittelbarkeit ein Bestehen gegen das Wesen bleibt, obwohl ihm dafür jede geltungstheoretische Grundlage fehlt und der Schein eigentlich unmittelbar sowie vollständig als Selbstaufhebung im Wesen verschwinden müsste. Keines der beiden Verhältnisse (Widerspruch/Integration) vermag sich dabei zu vollständiger Konsequenz seiner Struktur auszubilden, weshalb ihre zentrale Koinzidenz eben diese gemeinsame logische Inkonsequenz bleibt. Der Begriff des Scheins gestaltet derart das logische Zögern an sich als gestaltgewordene Unterbrechung des Vorgangs der Übersetzung von Gehaltsbestimmungen in Formbestimmungen der Logik. Mit anderen Worten: Wo die Form des Scheins (seinshaftes

Modelle von Dialektik sind so von einem existenziellen Ton und einer Vernichtungsangst getragen, die sie ebenfalls im Rahmen der Entwürfe des Deutschen Idealismus einzigartig machen. In einem Brief an seine Verlobte Wilhelmine von Zenge vom 30. November 1800 findet Heinrich von Kleist ein beeindruckendes Bild für diese negative, tragische Dialektik des Individuellen und des Allgemeinen. Kleist berichtet darin, wie er eines Abends in Würzburg spazieren geht und durch das gewölbte Stadttor tritt, um sich zur Stadt hin umzublicken. „Als die Sonne herabsank war es mir als ob mein Glück untergienge“, heißt es, und dann weiter: „Warum, dachte ich sinkt wohl das Gewölbe nicht ein, da es doch keine Stütze hat? Es steht, antwortete ich, weil alle Steine aufeinmal einstürzen wollen“. (Heinrich von Kleist: Sämtliche Werke und Briefe, hg.v. Klaus-Müller Salget u. a., Bd. 4: Briefe von und an Heinrich von Kleist 1793-1811, hg.v. Klaus-Müller Salget und Stefan Ormanns, München 1997, S. 159) Gerade in ihrem bestimmten Widerspruch zueinander halten sich die gegeneinander stürzenden Steine im Ganzen; sie bestreiten sich und gewinnen gerade dadurch Halt. Kleist selbst zieht aus diesem Bild den Trost des Gehaltenwerdens selbst im schlimmsten Moment unglücklichen Verworfenseins im Leben. Das Bild sagt: Weil alle Steine zugleich einstürzen wollen, halten sie sich im Fallen gerade aufrecht. Die Permanenz des Negativen sorgt hier für die Stabilität des Fortgangs. 
Bestehen) sich nicht dem Gehalt des Scheins (vollständige Negation des Seins im Wesen) angleicht, also diesen Übergang unterbricht, tritt eine Verzögerung des ,normalen' logischen Fortgangs der üblichen Form-Inhalt-Einheit ein. Im Schein steht das prozedurale Leben der Logik für einen logischen Moment still, weil er gleich einer Autoimmunreaktion des logischen Körpers die vollständige Aufhebung der überwundenen seinslogischen Grammatik in das Wesen zwar nicht vollständig aussetzt, aber auch nicht vollständig zur Geltung bringt.

Dadurch öffnet sich für das vollständig im Wesen aufgehobene Sein eine Art logisches Zeitfenster, in welchem es selbst noch mit seiner Auslöschung konfrontiert wird: Und die Frage stellt sich nun, welche Funktion diese aufwändige logische Prozedur Hegels, die einmal mehr von der auch prozeduralen Komplexität und Vielfältigkeit der Logik zeugt, ermöglicht. Denn es reicht nicht aus, auf den zutreffenden Umstand hinzuweisen, dass der Schein mit der eben entwickelten Grundstruktur erstmals in der Logik überhaupt die reine Wesensform selbstbezüglicher Negativität - allerdings auf noch unwahre, weil nicht-wesentliche und inkonsequente Weise - etabliert. Denn entscheidend dabei ist eben der Umstand, dass dieser Ursprung absoluter Negativität im Schein gerade darin seine Funktion und Geltung findet, sich dem all-inklusiven Aufhebungsgeschehen des Wesens bzw. in das Wesen zu widersetzen. Der Schein fungiert als und verwirklicht ein logisches Zwischen-Sein: Gegen die Verabsolutierung des teleologischen Mechanismus' der voranschreitenden, bestimmt-negativen Aufhebung, wie er in der „absoluten Kontextualität“80 bzw. der „absoluten Relationalität“ des Wesens erweitert und durch seine Verinnerlichung als Selbstbezüglichkeit des Wesens gänzlich von äußerlichen Widerständen befreit wird, verkörpert der Schein logisch ein Prinzip, das Hegel selbst zutreffend mit dem Terminus „Rest“ belegt (GW 11, S. 246). Ein „Rest“ ist ein nicht verwertbarer Überschuss, ein nicht weiter prozessierbares Übriggebliebenes, das sich den Operationen und Normen der Verarbeitung nicht fügt und ihnen folglich entgeht, sodass der Arbeitsprozess an ihm nicht vollständig durchgeführt wird. Der Rest ist das, was in dem Arbeitsprozess, in dem er entsteht, nicht weiter verarbeitet werden kann. Zugleich aber ist ein Rest auch etwas, das nicht einfach ignoriert werden kann, dessen Symbolwert darin liegt, als Markierung des Unterschieds zum verfehlten Ganzen weiter Geltung zu besitzen und Beachtung zu verdienen - wenn diese auch einzig darin liegt, nicht übersehen werden zu können. Der Schein bei Hegel vertritt

80 „Der Begriff absoluter Kontextualität zielt in erster Annäherung darauf, daß es für ein Denken, das sich intern zu organisieren (verstehen/begründen) sucht, nichts gibt, was eine äußere Grenze darstellen könnte: weder unmittelbare Empfindungen noch erste, selbstevidente Grundsätze oder letztinstanzliche (dogmatische) Entscheidungen.“ (Gerhard Gamm: Der Deutsche Idealismus. Eine Einführung in die Philosophie von Fichte, Hegel und Schelling, Stuttgart 1997, S. 109) 
dahingehend ein „Rest“ an unaufhebbarer seinslogischer Äußerlichkeit überhaupt, wie sie sich auf doppelte Weise - als formale Äußerlichkeit der Gedankenbestimmungen gegeneinander wie als inhaltliche Äußerlichkeit des als Sein gemeinten unmittelbaren „Realen“ gegenüber der Innerlichkeit des Wesens - im Sein als Grundprinzip geltend gemacht hatte. Im Schein besetzt das Sein eine Doppelstelle in Bezug auf das Wesen (außerhalb und innerhalb), die gemäß der genetischen Normativität der Logik gewissermaßen undenkbar, in jedem Fall in äußerlicher Betrachtung regelwidrig ist: Als eigentlich bereits vollständig aufgehobene Form kategorialer „Bestimmtheit überhaupt“ hat der Schein seine „Nichtigkeit [...] im Wesen“ (Hervorhebung C.W., GW 11, S. 246), d.h. das in ihm sich überlebende Sein wird als Schein konfrontiert mit seiner aktuellen, rechtmäßigen Gegebenheitsform als aufgehobenes Moment im Wesen, dem es als Monument seines Vergangenseins aktuell zugleich immer noch gegenübersteht. Im logischen Zögern des Scheins erhält sich in der Form der rhythmischen Wiederkehr, wie sie sich als Pendelbewegung seit dem Anfang der Wesenslogik über die Stufe des Unterschieds von Wesentlichem und Unwesentlichem angefangen hatte auszubilden und im Schein kulminiert, das Sein als unauslöschbare logische Erinnerung. Auch dieser Aspektkomplex kann übrigens wiederum in historischer Perspektive, d.h. innerhalb der Konstellation des Deutschen Idealismus, verortet werden: Hegel aktualisiert hier nämlich, mit entscheidenden Änderungen, den kantischen Unterschied von „logischem Schein“ und „transzendentalem Schein“ aus der Kritik der reinen Vernunft, indem er vor allem das Konzept des Letzteren seinem Scheinbegriff zugrunde legt. In der Einleitung zur „Transzendentalen Dialektik“, der zweiten Abteilung der „Transzendentalen Logik“, heißt es bei Kant:

\footnotetext{
Der logische Schein, der in der bloßen Nachahmung der Vernunftform besteht, (der Schein der Trugschlüsse,) entspringt lediglich aus einem Mangel der Achtsamkeit auf die logische Regel. So bald daher diese auf den vorliegenden Fall geschärft wird, so verschwindet er gänzlich. Der transzendentale Schein dagegen hört gleichwohl nicht auf, ob man ihn schon aufgedeckt und seine Nichtigkeit durch die transzendentale Kritik eingesehen hat. Eine Illusion, die gar nicht zu vermeiden ist, so wenig als wir es vermeiden können, daß uns das Meer in der Mitte nicht höher erscheine, wie an dem Ufer, weil wir jene durch höhere Lichtstrahlen als dieses sehen, oder noch mehr, so wenig selbst der Astronom verhindern kann, daß ihm der Mond im Aufgange nicht größer scheine, ob er gleich durch diesen Schein nicht betrogen wird. (KrV, B $353 \mathrm{f}$.)
}

Deutlich ist hier der Anknüpfungspunkt Hegels an Kant, der sich in einer begrifflichen Strukturgleichheit seines Scheinbegriffs zum „transzendentalen Schein“ äußert: Der Schein verschwindet wie der „transzendentale Schein“ nicht, obwohl er im Prozess des Übergangs vom Sein in das Wesen einen Inhalt ver- 
körpert wie bezeichnet, der durch diesen Übergang bereits (ebenfalls wie im „transzendentalen Schein“) als nichtig entwickelt worden ist, also quasi als Schein bereits durchschaut worden ist. Auffällig ist an dieser Stelle bei Kant zudem die zentrale Stellung der Termini „Nichtigkeit“ und „transzendentale Kritik“, die - natürlich objektiviert bzw. entsubjektiviert (im Sinne transzendentaler Subjektivität) - bei Hegel wiederkehren. Vor diesem Hintergrund sind es aber vor allem die Änderungen Hegels gegenüber Kant, die dem Scheinbegriff sein besonderes Gewicht verleihen. Dabei ist die entscheidende Modifikation bei Hegel nicht einmal die bereits angesprochene Evakuierung des Scheins aus den Gefahrenzonen transzendentaler Subjektivität, wie sie sich bei Kant noch in seinen analogischen Beispielen zeigt, die den Schein nämlich allesamt als Ereignis der subjektiven Wahrnehmung diskutieren; denn diese Modifikation gilt ja sozusagen generell für die ,Übersetzung، der kantischen Logik in die hegelsche. ${ }^{81}$ Entscheidend ist vielmehr Hegels Neubewertung, die damit einhergeht und dem Täuschungs- bzw. Illusionsgedanken des Scheins bei Kant gegenläufig positioniert ist: Im Gegensatz zu Kant erhält der Schein nicht nur einen „objektiven“ Grund im logischen Geschehen selbst und wird von der Wahrnehmungs- und Denkleistung transzendentaler Subjektivität entkoppelt, sondern seine unaufhebbare Artikulation eines Nicht-(mehr)-Wahrens (des Seins) wird als notwendige Funktion des aktuellen Stands der Wahrheit (des Wesens) - und nicht als bloß notwendiges Übel sinnvoller Vernunftfunktionen - verstanden. Die Dauerhaftigkeit des Falschen im Schein bringt gerade nicht die Hartnäckigkeit überwundener kategorialer Muster zum Ausdruck: Das würde nur dann gelten, wenn deren Defizienz nicht bereits vollständig erkannt worden und somit Teil eines sich durch Verdunkelung erhaltenden logischen Scheins wäre. Es ist deshalb entscheidend, dass Hegel betont, wie sich das Sein im Schein trotz der bereits vollständigen Aufdeckung und begrifflichen Durchdringung seines Aufgehobenseins restituiert, um eben einer Interpretation vorzubeugen, die den Weg über den Schein als bloßen Ausdruck ungenügender Reflexionstätigkeit kennzeichnet.

Der Schein ist die iterative logische Operation schlechthin: indem er den Punkt der reinen Wiederkehr des Negierten als Negiertes, das Vorhandensein eines Verschwundenen (des Seins) als Verschwundenes (und nicht als durch die Aufhebung immer schon integriertes, aktuelles) markiert und kategorial festhält. Um diese Denkfigur logischer Zeitlichkeit ${ }^{82} \mathrm{zu}$ verstehen, ist des Öfteren bereits in

81 Vgl. Taiju Okochi: Ontologie und Reflexionsbestimmungen. Zur Genealogie der Wesenslogik Hegels, Würzburg 2008, S. 21.

82 Die Zeitlichkeit der Logik liegt nicht allein in ihrer Form der reinen Abfolge als zeitlicher Aspekt der A-Reihe (nach John M.E. McTaggert: „The Unreality of Time“, in: The Philosophy of Time, hg.v. Robin Le Poidevin, Murray MacBeath, Oxford 1993, S. 23-35), gemäß deren Gesetz des 
analogischer Weise - und begründet in Hegels Sprechweise vom ,zeitlos vergangene[n] Seyn“ (GW 11, S. 241) - auf die reale Zeitlichkeit des Erinnerns zurückgegriffen worden; etwa, wenn Iber den Schein mittels des Unterschiedes von Vergangenem und Niegewesenem erklärt: „Dem liegt nun keine denkerische Inkonsistenz zugrunde, sondern vielmehr die Behauptung, daß das Vergangene als Vergangenes präsent bleibt und sich somit vom Niegewesenen unterscheidet. Das Sein gerät im Schein aus der Vergangenheit ins Präsens. Genauer: es gerät aus der Vergangenheit ins Präsens, ohne darin nicht mehr vergangen zu sein. "83 Es ist allerdings weiterhin $\mathrm{zu}$ beachten, dass im Schein nicht bloß iterativ das Sein in seiner bloßen Leerform, als paradoxe Rückkehr des Falschen auf falsche Weise trotz seiner Falschheit, zum Ausdruck kommt. Dies wäre eben jene Interpretation des Scheins, die dessen Stufe zu Anfang der Wesenslogik als ein reines retardierendes Moment der logischen Entwicklung betrachtet, das für sich selbst keinerlei begrifflichen Eigenwert besitzt, sondern quasi nur die „wahre“ Entwicklung verzögert. Demgemäß heißt es bspw. bei Iber: „Der Schein ist die leere Stelle, dieses rein gedankliche Vakuum, welches das Sein hinterließ, als es ein Anderes als das Wesen zu sein nicht mehr vorgab. “84

Demgegenüber ist aber der regenerative Aspekt des Scheins als nähere Bestimmung seiner grundsätzlich iterativen Operationsweise unbedingt ernst $\mathrm{zu}$ nehmen, damit der positive Effekt des „Scheins überhaupt“ in den Blick kommen darf. Im Schein wird das Sein nicht nur als Falsches wieder(ge)holt, sondern auch in bestimmten Aspekten seines einstigen Geltungsanspruchs wiederhergestellt. Das Sein als Terminus für die Art seinslogischen Bestimmens bleibt natürlich verschwunden und grundsätzlich sowie vollständig im Wesen gänzlich aufgehoben; die bestimmte Negation des Seins im Wesen und damit die Geltungsmacht des Wesens für den Stand der logischen Wahrheit darf an keinem Punkt revidiert

Auseinander-Hervorgehens die Kategorien gemäß dem Prinzipkomplex der Aufhebung (der analog selbst eine solche Entwicklung durchmacht) allein zu denken sind. Auch der Aspekt der BReihe nach McTaggert greift hier: Denn die Kategorienentwicklung kennt auch eine Entsprechung der Aspekte von Vergangenheit und Zukunft dergestalt, dass es für jede Gedankenbestimmung ein logisches Moment gibt, in Bezug auf welches sie der Möglichkeit des Wahren nach angemessen ist (ihre logische Gegenwart), und viele weitere logische Momente, zu denen sie dem Wahren nach unangemessen ist (ihre logische Zukunft), sowie solche, zu denen sie noch undenkbar ist (ihre logische Vergangenheit). Anders gesagt: Jede Entwicklungsposition in der Logik lässt sich so beschreiben, dass vergangene Bestimmungen ihrer Norm des aktuell Wahren unangemessen und kommende Bestimmungen mit ihrer je weiterentwickelten Norm des Wahren undenkbar sind. Erst durch eine solche Konstruktion wird es möglich, dass in Bestimmungen wie dem „Schein“ die logische Gleichzeitigkeit des Ungleichzeitigkeiten wichtige semantische Effekte auslösen kann. 83 Iber: Metaphysik absoluter Relationalität, S. 71.

84 Iber: Metaphysik absoluter Relationalität, S. 70. 
werden. Zugleich aber kehrt in der Leerform des Seins als Schein, d.h. in der Wiederkehr des Überwundenen und Verschwundenen als Überwundenes und Verschwundenes, ein anscheinend unabweisbarer Geltungsanspruch wieder, welcher mit der Äußerlichkeit seinslogischen Bestimmens, wie sie als reine Form selbst in ihrer vollständigen Negation im Schein überdauert, verbunden ist: Ausgedrückt findet sich dies schon allein in der logischen Eigenzeit, die dem erneuten Auftauchen des Scheins in der Logik zugestanden wird, und die aufgrund ihrer Ausnahmestellung in der Logik bereits an sich selbst bedeutungstragend ist. Es ist entscheidend zu betonen, dass entgegen mancher Lesarten des Schein-Abschnitts ${ }^{85}$ dieser in der hier vorgeschlagenen Perspektive nicht so verstanden werden darf, als sei in ihm der nicht genügend durchargumentierte Übergang in das Wesen als einfache Defizienz der begrifflichen Erschließung des Wesens gedacht: So nämlich wird der Schein zu einer bloßen Verzögerung des Voranschreitens bestimmter Negation des Seins, deren Funktion einzig darin liegen kann, am Grad des Widerstands des zu Überwindenden (des Seins) proportional die Größe und Kraft des gegenwärtigen Stands logischer Wahrheit (des Wesens) hervortreten zu lassen. Der Schein aber ist nicht (bloß) platonische do$x a^{86}$ : Denn damit würde er wieder nur zu einer Variante des kantischen „logischen Scheins“ - und somit eben den Charakteristika widersprechen, die ihn klar dem „transzendentalen Schein“ zuordnen. Natürlich soll dem grundsätzlich negativen Charakter des Scheins nicht widersprochen werden: Die strenge Zuordnung, die Theunissen in seiner Interpretation der Logik für das „Verhältnis von Schein und Kritik“87 entwickelt, kann und darf grundsätzlich nicht infrage gestellt werden. Gerade wenn man wie Theunissen den Blick auf die gesamte Logik richtet und den Schein nicht primär als Gedankenbestimmung einer beschränkten logischen Position zu Anfang der Wesenslogik, sondern vor allem als in der ganzen Logik von Anfang bis Ende präsentes Prinzip der bloßen „Unwahrheit“"88 versteht, die kategorial das „intentionale Korrelat der Kritik“ ${ }^{* 89}$, d.h. der bestimmten Negation, ausmacht, ist dies völlig überzeugend. Es geht hier deshalb nicht um ein Entweder-oder, sondern darum, eine zusätzliche Schicht der logischen Funktion des Scheinbegriffs hervorzuheben, die zur negativistischen Lesart Theunissens in ein Ergänzungs-, nicht in ein Widerspruchsverhältnis tritt. Der Unterschied beider Lesarten ist schon deshalb kein krisenhafter, konfligierender, weil sie, wie bereits

85 Vgl. bspw. Iber: Metaphysik absoluter Relationalität, S. 14; Henrich: Hegels Logik der Reflexion, S. $106 \mathrm{f}$.

86 Vgl. Theunissen: Sein und Schein, S. 72.

87 Theunissen: Sein und Schein, S. 73.

88 Theunissen: Sein und Schein, S. 71.

89 Theunissen: Sein und Schein, S. 72. 
angesprochen, mit unterschiedlichen Auflösungen bzw. Reichweiten des Verstehens gekoppelt sind: bei Theunissen der Blick auf Prinzipien und logische Gegenstände der gesamten Logik, in meiner Interpretation der Blick auf die besondere logische Position nur im Übergang von der Seins- zur Wesenslogik. Ebenso wenig besteht ein krisenhafter Widerspruch zwischen der Idee einer Kontinuität des Falschen (des Seins) im Schein, die im Ausdruck „Rest“ ebenfalls insinuiert ist und ein Verständnis zu unterstützen scheint, welches auf die ungenügende Durchführung der Aufhebung des Seins abhebt, und meiner iterativen Lesart einer bewusst inszenierten Wiederkehr des Seins in gestaffelten Pendelbewegungen: also zwischen dem Verständnis, dass das Sein nie richtig aufgehoben worden und deshalb im Schein als Rest anwesend ist, und dem Verständnis, dass das Sein in gewisser Hinsicht bereits vollständig aufgehoben wurde und deshalb im Schein (wiederum nur in gewisser Hinsicht) wiederkehrt. Der Begriff der Hinsichtnahme ist hier eben entscheidend: Hegels Logik ist ein derart multidimensionales Gebilde, dass sich oftmals verschiedene Funktionskontexte überlagern. In Hinsicht darauf, dass das Wesen zu Anfang der Wesenslogik nicht gänzlich und dauerhaft den gesamten logischen Raum eingenommen hat, kann von einem homogenen Weiterbestand des Seins in den Stufen Wesentliches/Unwesentliches und Schein gesprochen werden. In Hinsicht darauf, dass das Wesen als seine notwendige und hinreichende Voraussetzung die vollständige Aufhebung des Seins bereits hinter sich hat („Das Wesen ist das a uf g e ho be ne Se y n“, GW 11, S. 245) und seinem minimalen logischen Begriff nach eigentlich nichts anderes als die bereits vollzogene Aufhebung des Seins ist ${ }^{90}$, muss dort, wo im Schein irgendein Geltungsanspruch aus dem Bereich des Seins konstatiert wird, der aber in der Gegenwart des Wesens gar nicht mehr existieren kann, eben von einer partiellen, punktuellen und ephemären Wiederkehr gesprochen werden. Es macht die Faszination und Komplexität des Abschnitts zum Schein aus, dass Hegel diese Perspektiven überlagert, um verschiedene logische Funktionen mit unterschiedlicher Reich-

90 Diese Dopplung drückt sich erstens darin aus, dass im Schein das Sein vollständig und komplett (und nicht nur unvollständig und teilweise) verneint ist, also gänzlich aufgehoben vorliegt, denn nur so ist die Leerheit des Scheins als sein kategorialer Gehalt überhaupt möglich. Hegel macht dies ausdrücklich klar in seinen Bestimmungen „unmittelbares Nichtdasein“ und „reflektierte Unmittelbarkeit“. Damit aber bringt der Schein zum Ausdruck, dass das Sein eigentlich bereits vollständig im Wesen verschwunden, die Aufhebung also umfassend zu denken ist. Zweitens zeigt sie sich dann darin, dass der Maßstab und Motor der zweiten Aufhebung des Seins als Schein in der zweiten Hälfte des Abschnitts „B. Der Schein“ eben jene eigentlich immer schon gänzlich vollzogene Aufhebung des Seins im Wesen ist: Nur in unter dieser Voraussetzung findet die zweite Aufhebung des Scheins im Wesen überhaupt ihre Möglichkeit, nämlich als Erinnerung an das bereits vorliegende Wesen. So argumentieren auch Iber (Metaphysik absoluter Relationalität, S. 229-231) und Henrich (Hegels Logik der Reflexion, S. 108). 
weite und Umfang zusammenzustellen, ohne diese sich gegenseitig in die Quere kommen zu lassen. Was als Geltungsanspruch des vollständig verneinten Seins im Schein wiederkehrt - und was gerade wegen seiner prinzipiellen Aufhebbarkeit im Wesen als iterativ-regenerativ nicht-aufgehoben sich artikuliert -, muss eine klare positive Funktion für das Wesen haben und nicht bloß ein Defekt seines verzögerten Erarbeitungsprozesses sein. Der so inszenierte Rückstoß seinshafter Äußerlichkeit und Unmittelbarkeit auf der Stufe ihrer geltungstheoretisch eigentlich vollständigen Haltlosigkeit stellt einen bestimmten Anspruch auf logische Verantwortlichkeit her, den das Wesen von nun an zu beachten hat und der auch den Schlüssel zu meiner realitätskategorialen Lesart der Reflexionsformen darstellt. Mit anderen Worten: Das Wesen erhält durch die Haltbarkeit des Seins im Schein die Verantwortung zugesprochen, bestimmte Bestimmungen des Bestimmens aus dem Sein auch dort weiterhin zu beachten, wo es diese in seinem Sinn bereits verwandelt hat. Das Wesen muss mit dem Rest an Seinsanspruch umgehen, der weder ganz im Wesen verschwindet, noch ganz als Anderes zum Wesen bestehen bleibt. Gerade die zweite Hälfte des Scheinabschnitts, indem sie das im Schein verkapselte negierte Sein erneut - und nun wirklich restlos - in das Wesen integriert, führt diese Konsequenz weiter aus. 